CAHIERS DE

NARRATOLOGIE

\section{Cahiers de Narratologie}

Analyse et théorie narratives

$30 \mid 2016$

Street Art 2

\title{
Le « street art » est-il fini ?
}

Notes pour une lecture esthétique et critique de l'art urbain

\section{Vittorio Parisi}

\section{(2) OpenEdition}

Journals

Electronic version

URL: http://journals.openedition.org/narratologie/7511

DOI: 10.4000/narratologie.7511

ISSN: 1765-307X

Publisher

LIRCES

\section{Electronic reference}

Vittorio Parisi, « Le « street art » est-il fini ? », Cahiers de Narratologie [Online], 30 | 2016, Online since 28 July 2016, connection on 01 May 2019. URL : http://journals.openedition.org/narratologie/7511 ; DOI : 10.4000/narratologie.7511

This text was automatically generated on 1 May 2019.

Article L.111-1 du Code de la propriété intellectuelle. 


\title{
Le « street art » est-il fini?
}

Notes pour une lecture esthétique et critique de l'art urbain

\author{
Vittorio Parisi
}

1 «FAME Festival est mort. Ce que vous êtes, nous l'étions. Ce que nous sommes, vous le serez. Mais toujours pis et $\operatorname{tard}^{1}$. »

2 Nous pouvons lire ces mots sur l'ancien site du FAME Festival, qui a eu lieu à Grottaglie, une petite ville dans l'Italie du Sud, à partir de 2008 jusqu'à 2012. Ces mots dramatiques, ayant un air décidément lapidaire et solennel, ont été écrits par le fondateur et organisateur du festival, Angelo Milano. FAME était un festival que j'ai eu la chance d'observer d'assez près, et de visiter à plusieurs reprises durant les années de l'université, ma ville d'origine n'étant qu'à une centaine de kilomètres de Grottaglie. Et cela a été sans doute une chance parce que, malgré sa "mort», le FAME demeure à ce jour l'une des expériences les plus intéressantes et enrichissantes liées à ce milieu, ou mieux ce "monde ", communément appelé «street art». Durant sa vie, courte mais intense, le FAME a su capturer l'attention des médias spécialisés les plus importants à travers un site comme Grottaglie, sur la limite entre l'urbain et le rural, et son union avec une programmation artistique de grande qualité, contribuant à la formation et à la célébrité de beaucoup d'artistes (par exemple JR et Vhils) et développant un commerce international d'œuvres d'art.

3 Mais qu'est-ce qui aurait poussé son organisateur à suspendre une expérience dont le succès était loin de faner ? À arrêter un festival dont l'identité était très forte et le format pratiquement impossible à imiter? Le cas du FAME Festival nous permet d'appeler en cause l'idée de « fin du street art ».

4 Avant de rentrer dans le vif du sujet, je préfère éclaircir l'usage que j'entends faire de l'expression street $\mathrm{art}^{2}$, en raison de plusieurs doutes terminologiques. Street art est, en effet, une formule porteuse de plusieurs contradictions : en nous limitant à son signifié strictement littéral d'art de/dans la rue, ou plus généralement de/dans l'espace urbain, d'un coté nous serions contraints d'en exclure catégoriquement les toiles ou les installations que les « street artistes » exposent à l'intérieur des galeries; d'un autre, nous pourrions au contraire y inclure les monuments, les installations d'art public ou des 
fresques urbains tels que les trompe-l'œil, autrement dit des manifestations produites par des milieux artistiques complètement différents. Des locutions alternatives à street art ont été récemment formulées précisément pour esquiver ce malentendu. Rafael Schacter a notamment proposé «independent public art» (art public indépendant $\left.{ }^{3}\right)$, Ulrich Blanché «self-authorized art» (art auto-autorisét), pour marquer la différence avec d'autres types d'art urbain issus d'un cadre institutionnel. Cependant, l'efficacité de telles formules s'arrête justement là où ces artistes publics " nés " indépendants ou " autoautorisés » répondent aux logiques du marché de l'art (par exemple en exposant dans des galeries ou en réalisant des interventions sur commande), ou collaborent avec des institutions telles que les musées ou les administrations citoyennes. Ainsi, je pense que rechercher la «formule parfaite » soit une mission vouée à l'échec, puisque nous ne nous trouvons pas face à un genre artistique, ni à un style ou à une tendance, ni à un mouvement ou à une école, mais à un ensemble hétérogène et pluraliste de tout cela : un véritable monde de l'art au sens dantien du terme ${ }^{5}$, alternatif au monde de l'art soi-disant « institutionnel», et qui en même temps est dans un rapport d'intersection avec ce dernier. Une fois cette conscience acquise, le problème des mots devient secondaire, et nous pourrions assumer la même politique de praticité que Hegel décida d'assumer quand, dans l'introduction à ses Leçons de 1828, s'interrogeait sur la pertinence du mot " esthétique »:

[...] le terme esthétique n'est pas tout à fait celui qui convient. On a encore proposé des dénominations : « théorie des belles sciences », « des beaux arts », mais elles ne se sont pas maintenues, et avec raison. On a également employé le terme de «callistique» [...] Nous allons donc nous en tenir au terme Esthétique, non parce que le nom nous importe peu, mais parce que ce terme a reçu droit de cité dans le langage courant, ce qui est déjà un argument sérieux en faveur de son maintien ${ }^{6}$.

Une fois accepté donc de me servir de l'expression street art, malgré ses ambiguïtés, en vertu de son usage dans le langage courant, je vais faire front à un deuxième doute : quel intérêt, en fin de compte, pourrait avoir le fait de parler, encore une fois, de « fin de l'art »? Effectivement nous savons jusqu'à quel point l'histoire de la pensée, de la théorie et de l'historiographie artistique abonde d'affirmations apocalyptiques concernant l'«état de santé » ou de légitimité de l'art. De son bannissement de la république imaginée par Platon, en raison de sa nature trompeuse donc dangereuse, à sa toute première déclaration de mort déclaré par Vasari, là où l'idée moderne d'historiographie artistique venait tout juste d'être fondée : «Tels que les corps humains, les arts naissent, grandissent, vieillissent et meurent ${ }^{7} »$. De l'inéluctable destin tracé par Hegel avec sa célèbre sentence ("L'art est pour nous quelque chose du passé8 $»)$ jusqu'aux élaborations les plus récentes de ce même constat, proposées en 1984 par Arthur Danto avec l'essai The End of Art $^{9}$.

6 Face à une telle panoplie de voix illustres et à un thème aussi recourant, je me demande si parler de «fin du street art» ne serait, somme toute, qu'une minauderie, et non pas l'occasion d'apporter une lecture véritablement esthétique et critique du phénomène street art, c'est qui est d'ailleurs l'objectif principal de ce texte. À ceci s'ajoute le constat qu'un sujet tel que le street art fait l'actualité de nombreux rencontres, expositions, colloques et publications dont celle-ci: ne serait-elle en soi une raison suffisante à déclarer le street art toujours vivant, bien loin d'une fin, réelle ou hypothétique que ce soit?

7 Tout en sachant qu'il n'y a jamais eu et, peut-être, il n'y aura jamais une véritable fin à l'art, et que parler de cela n'est qu'un artifice théorique, cette fin de l'art a été souvent 
annoncée à des moments où l'art avait l'air d'être, au contraire, en pleine effervescence. Toutefois en même temps, les conditions qui avaient permis l'apparition du phénomène artistique observé avaient atteint, ou étaient en train d'atteindre, leur propre épuisement.

Ainsi avec Hegel le modèle «biologique » qui, de Vasari à Winckelmann, avait caractérisé le pivot de toute conception historiographique classique, n'était désormais plus adéquat. L'historien de l'art Hans Belting (auteur de l'essai «L'histoire de l'art est-elle finie?» dont le titre de cet article s'inspire ${ }^{10}$ ) voit dans l'idée de «fin de l'art » développée par le philosophe le "symptôme d'une nouvelle compréhension de l'art ${ }^{11}$ ». Cette "nouvelle compréhension » était une véritable nécessité à l'époque où le romantisme commençait à balayer cet esprit classique dont Winckelmann fut un interprète exquis. L'histoire de l'art a par la suite connu d'autres occasions où il fallait une "nouvelle compréhension », où il fallait trouver d'autres mots, de nouveaux critères, des nouveaux prédicats pour interpréter son évolution, qui se faisait de plus en plus fragmentée et caractérisée, comme l'aurait dit Benedetto Croce un siècle plus tard, par une évidente « insularité ${ }^{12}$ ».

9 Arthur Danto a été le dernier à se charger de cette quête, en essayant de lui donner une solution définitive. Il le faisait au moment où Andy Warhol proposait une copie parfaite de la boîte de lessive Brillo comme œuvre d'art, qui se rajoutait à la composition de 4'33" de silence par John Cage et à l'exposition du vide par Yves Klein. C'est le moment où tout objet, même un objet immatériel ou relevant du banal, pouvait être investi d'une aura artistique. Et c'est aussi le moment où tout style, tout genre, tout contenu, toute forme cohabitent et s'hybrident. Danto parle clairement de "pluralisme », et il y voit la cause d'une nouvelle « fin de l'art ${ }^{13}$ ». Ce pluralisme serait donc le grand détour qui a fait bouger les contours de l'histoire de l'art telle qu'on l'avait connue auparavant: c'est-à-dire comme une succession linéaire de périodes et de mouvements bien définis.

10 Et si l'on devait donc s'interroger sur la " fin du street art ", quels seraient les détours qui l'auraient rendue possible? Quelles, les conditions originaires à avoir atteint un épuisement ? C'est ici qu'on revient au cas du FAME.

11 FAME naît comme festival «d'art public ». Ainsi l'a conçu son organisateur, qui a toujours refusé catégoriquement l'emploi de l'expression street art. Interviewé par le magazine italien Artribune, il dit :

Je ne me suis jamais occupé de street art, la seule chose que j'aimais faire, c'est d'inviter mes amis ici pour foutre le bordel. Le street art n'existe que dans les livres en anglais, ou dans les médias comme celui-ci. Ni moi ni mes amis n'avons jamais eu rien à foutre avec ces conneries ${ }^{14}$.

12 L'esprit du FAME est proche d'une certaine culture punk que son fondateur, musicien dans un groupe screamo, n'hésite pas à revendiquer. Cet esprit est également incarné par la culture DIY (Do It Yourself - fais-le toi même) : le festival ne recherche aucun soutien public ni institutionnel, aucune sponsorisation privée, les artistes sont pour la plupart des autodidactes et leurs interventions ne sont pas autorisées. L'objectif du FAME et de ses invités c'est littéralement de «fare casino » (foutre le bordel), en faisant quelque chose de nouveau et de jamais vu dans un petit village provincial de l'Italie du sud. Au début cela a naturellement produit des contrastes avec les autorités locales et avec une partie des citoyens de Grottaglie, et durant les premières éditions le FAME a rencontré plusieurs obstacles: un grand mural d'Ericailcane a été effacé par la Mairie pour protéger le patrimoine architectural de la ville de Grottaglie ${ }^{15}$. 
Cependant, assez rapidement cette «aura » de vandalisme qui semble façonner l'esprit originaire du festival commence à rencontrer le soutien des médias :

Les premières années c'était beaucoup mieux, car le festival engendrait des contrastes et cela était très amusant. Ensuite, vue la renommée que les artistes invités étaient en train de gagner, la presse et internet ont commencé à diffuser la rumeur que FAME était un événement qu'il fallait soutenir. Conséquemment les contrastes ont disparu et le tout s'est affaibli dans un consensus général, littéralement dicté par les médias: plus l'article était con, plus il conférait de l'autorité au festival ${ }^{16}$

Voilà enfin la décision de « faire mourir » le festival :

À la fin de la dernière édition tout était devenu assez simple : peindre partout, faire des vidéos, défoncer des trucs même sous le regard des autorités. Nous avons eu beau essayé de faire des choses moches et provocatrices, mais nous n'avons gagné que de sourires et de la bienveillance. La friction des origines avait disparu [...] Partout en Italie et en Europe les festivals se sont multipliés, devenant tous identiques et interchangeables. Les administrations et les politiciens ont commencé à financer leur petit festival et la consommation de l'observateur a commencé à se limiter à quelques clics sur Facebook. J'ai donc ressenti un manque total de sens en ce que nous étions en train de faire ${ }^{17}$.

La parabole du FAME Festival, de ses origines à sa mort proclamée, exemplifie très bien l'idée de « fin du street art » que le présent article veut communiquer. Lors que le street art gardait une dimension esthétique anti-décorative, que son âme était viscéralement liée à une certaine idée de "foutre le bordel » et qu'il devait tenir compte de la réaction contrariée et souvent répressive de l'autorité (comme s'il s'agissait d'une partie fondamentale et inaliénable de la pièce artistique, propre à l'œuvre et à sa valeur à la fois matérielle et performative), le street art avait le droit de revendiquer un véritable statut d'avant-garde, puisque ce statut ne pouvait être lié qu'à sa propre aura d'acte non réclamé et non autorisé.

Nous sommes face à une aporie : le FAME, vus les principes qu'il incarnait et en voulant rester fidèle à ces mêmes principes, ne pouvait qu'être voué à l'autodestruction. Encadrer un art indépendant, spontané, non autorisé et tumultueux sous la forme d'un festival c'est en soi une manière de le reléguer dans une cage, de contribuer à son institutionnalisation, donc à sa mort. Un festival est déjà en soi un moyen institutionnalisant, en tant qu'événement organisé, répondant à une logique à la fois expositoire et communicationnelle. Et avec leur vocation à joindre un public beaucoup plus vaste et transversal, les festivals ont joué, dans ce processus dégénératif, un rôle peut-être plus décisif que celui des galeries d'art.

Un cas également emblématique de cette aporie est représenté par l' « euthanasie » (on est toujours dans un vocabulaire où l'idée dominante est celle de fin ou de mort) que l'artiste italien Blu a voulu donner au grand mural qu'il avait réalisé en 2007 à Kreuzberg, à Berlin, l'effaçant le 19 décembre $2015^{18}$. Depuis quelques années, Kreuzberg, quartier symbole du Berlin underground, connaît une vague de spéculation foncière et de gentrification qui se doit en partie à la présence massive de street art. Blu, en commettant ce suicide artistique, en utilisant les mêmes moyens de censure qu'utiliserait une administration citoyenne pour protéger son patrimoine architectural ou une propriété privée, a commis un geste sans précédent : un geste engagé qui rend de la légitimité et de la fraîcheur à un art qui, autrement, ne serait plus qu'un simulacre. 
18 Tant de la fin du FAME Festival que du geste de Blu nous pouvons tirer surtout une leçon de conscience critique, une prise de conscience qui demeure assez rare dans un panorama qui se montre dominé, au contraire, par un enthousiasme assez plat et acritique. Cet enthousiasme se reflète, par exemple, dans la vague de «muralisation» des périphéries en Italie, notamment à Rome dans les quartiers de Tor Marancia ou de Tor Pignattara. Pourquoi devrions-nous considérer dangereuse l'action d'embellissement de quartiers-ghetto?

20 Les quartiers de logements sociaux en Italie, notamment ceux qui ont été bâti à partir des années 1950, sont souvent structurés comme des véritables ghettos, situés à l'extérieur du conglomérat urbain, dépourvus des services nécessaires comme les transports et les commerces, des endroits oubliés par les administrations, où la criminalité liée au trafic de drogue trouve son terrain d'action privilégié.

21 En juin 2014 j'ai organisé avec mon association culturelle Pigment Workroom un projet d'art urbain dans le quartier d'Enziteto à Bari, ma ville d'origine. Le titre du projet était sarcastique: Enziteto Real Estate $^{19}$ et avait pour but d'attirer l'attention de l'administration sur un quartier qui vit une situation sociale assez délicate, à travers sa transformation imaginaire en lieu de "promotion immobilière » ou de "village de vacances ", où tout le monde s'amuse grâce au pouvoir récréatif et embellissant du street art. Nous croyions lancer un message critique assez fort, intégrant d'une manière sarcastique la dialectique médiocre du street art comme moyen de requalification urbaine, sauf que la mairie de Bari nous a pris à la lettre : le maire et son adjoint à la culture ont à plusieurs reprises parlé de notre projet avec grand enthousiasme à travers leurs pages Facebook, en soulignant la valeur positive du street art, mais aucun délégué de la mairie s'est finalement engagé pour inaugurer une nouvelle et véritable discussion, pour redessiner le destin peu heureux d'Enziteto.

Derrière cette vague de muralisation se cache donc le risque que les administrations citoyennes emploient le street art en tant que moyen captivant et peu coûteux de liquider les véritables problèmes qui affectent les périphéries.

Ce vif enthousiasme pour la soi-disant valeur positive du street art est le même qui accompagne, depuis quelque temps, chaque nouveau coup de théâtre de Banksy, comme le plus récent Dismaland. Dans le cas de Banksy, cette valeur serait incarnée par une forme de street art prétendument engagé, altermondialiste et encore une fois très sarcastique. Les pochoirs ou les installations ironiques de Banksy seraient souvent le véhicule d'une satire féroce, ainsi que de fréquentes critiques contre le marché de l'art. Dismaland, en serait l'exemple parfait. Mais le problème, avec Banksy, c'est le même qui se pose pour la plupart des vedettes de la scène artistique contemporaine : face au récit incessant que les médias nous offrent de ses gestes, cela devient compliqué de s'apercevoir de son populisme déguisé en satire sociale, de l'écrasante banalité de ses affirmations, de l'incohérence qui se cache derrière la critique de ce même marché dont il tire continûment avantage ${ }^{20}$. L'art de Banksy est un art kitsch au sens benjaminien du terme, soit un art qui vise à produire un agrément instantané chez son récepteur ${ }^{21}$, dans ce cas à travers des langages immédiats, récréatifs, simulant ceux de la publicité, mais en même temps il s'agit d'un art qui dépasse cette même définition de kitsch. Ce kitsch de Banksy est à la foi autocomplaisant et adulateur : il trompe son observateur en lui faisant croire d'être face à quelque chose d'engagé et de critique, tout en conservant une certaine coolness, une esthétique qui est tout à fait subordonnée aux diktats du marché. 
l'intériorisation et le dépassement de la fin du street art se manifeste naturellement dans l'activité d'artistes comme Blu, ou Escif, ou dans le travail du duo napolitain Cyop \& Kaf qui, tout en restant indépendants et non autorisés (ils ne travaillent pas avec des galeries) et sans aucune revendication ou présomption d'amélioration ou d'embellissement, ont parsemé les Quartieri Spagnoli de Naples et la Città Vecchia de Taranto de créations éphémères et à l'échelle humaine, dans une démarche volontairement anti muraliste et anti monumentaliste ${ }^{24}$.

30 Ces types d'approche me font penser que, heureusement, bien que le street art semble avoir définitivement achevé sa fonction auparavant liée à une autonomie radicale, à une véritable indépendance, à une vocation à créer des chocs, malgré il soit aujourd'hui toute autre chose, je trouve qu'il peut encore, en certains cas, se révéler capable de modifier et de remettre en question le réel. De le transfigurer, de construire de nouvelles identités, 
de dire quelque chose de vraiment important et de le dire en pleine conscience de sa propre histoire.

\section{BIBLIOGRAPHY}

Belting, H. (2005) L'histoire de l'art est-elle finie ? Paris : Folio.

Benjamin, W. (1999) The Arcades Project. Cambridge : Harvard University Press.

Croce, B. (1907) Ciò che è vivo e ciò che è morto della filosofia di Hegel; studio critico. Bari: Laterza.

Cyop \& Kaf (2014) Taranto. Un anno in città vecchia. Napoli : Monitor.

Danto, A.C. (1964) «The Artworld ». In : The Journal of Philosophy, Volume 61, Issue 19. New York: American Philosophical Association.

Danto, A. C. (1986) The Philosophical Disenfranchisement of Art. New York : Columbia University

Press.

Danto, A.C. (1981) The Transfiguration of the Commonplace. Cambridge: Harvard University Press.

Escif (2015) Elsewhere. Valencia : Martin Impresores.

Foucault, M. (2001) Dits et écrits Tome I 1954-1975. Paris : Gallimard.

Hegel, G.W.F. (1944) Cours d'esthétique, Tome I. Paris : Aubier.

Morin, M., Mezache, S. (2015) Saeio Nolens Volens. Paris : Galerie P38, catalogue d'exposition.

Vasari, G. (1986) Le vite de' più eccellenti pittori, scultori ed architetti. Torino : Einaudi.

\section{NOTES}

1. Milano, A. (2013) FAME Festival è morto. http://www.famefestival.it/fame-festival-e-morto/ [13.11.15]

2. Uniquement pour des raisons pratiques je vais inclure dans cette formule le type d'art impliquant la réalisation des tags, et communément appelé "graffiti », « writing » ou " graffiti writing ".

3. Schacter, R. (2014) Ornament and Order. Graffiti, Street Art and the ParergoN. London : Routledge.

4. Blanché, U. (2015) « Qu'est-ce que le Street art? Essai et discussion des définitions ». In : Cahiers de Narratologie [Online], 29. Online since 31 January 2016, connection on 30 April 2016.

5. Danto, A.C. (1964) «The Artworld». In: The Journal of Philosophy, Volume 61, Issue 19. New York: American Philosophical Association, 571-584.

6. Hegel, G.W.F. (1944) Cours d'esthétique, Tome I. Paris: Aubier, 17-18.

7. « Come i corpi umani, le arti hanno il nascere, il crescere, lo invecchiare et il morire», Vasari,

G. (1986) Le vite de' più eccellenti pittori, scultori ed architetti. Torino: Einaudi, 132.

8. Hegel, G.W.F. (1944) Op. Cit., 18.

9. Danto, A. C. (1986) The Philosophical Disenfranchisement of Art. New York : Columbia University Press, 81-115. 
10. Belting, H. (2005) L'histoire de l'art est-elle finie ? Paris : Folio.

11. Ibidem : 35 .

12. Croce, B. (1907) Ciò che è vivo e ciò che è morto della filosofia di Hegel; studio critico. Bari : Laterza.

13. Danto, A.C. (1986) Op. Cit. ; Danto, A.C. (1964) « The Artworld». In : The Journal of Philosophy, Volume 61, Issue 19. New York : American Philosophical Association, 571-584 ; Danto, A.C. (1981) The Transfiguration of the Commonplace. Cambridge : Harvard University Press.

14. Tonelli, M. (18.05.2014) «Chiudere all'apice della notorietà. Angelo Milano racconta il Fame Festival ». In : Artribune. http://www.artribune.com/2014/05/chiudere-allapice-della-notorietaangelo-milano-racconta-il-fame-festival/ [13.11.15]

15. Ibidem

16. Ibidem

17. Ibidem

18. Henke, L. (19.12.2014) « Why we painted over Berlin's most famous graffiti ». In : The Guardian. http://www.theguardian.com/commentisfree/2014/dec/19/why-we-painted-over-berlingraffiti-kreuzberg-murals [13.11.15]

19. Brochure en ligne du projet : http://issuu.com/pigmentworkroom/docs/enziteto-real-estatecatalog [13.11.15]

20. Banksy (21.08.2015) Banksy: «'I think a museum is a bad place to look at art'». In: The Guardian. http://www.theguardian.com/artanddesign/2015/aug/21/banksy-dismaland-artamusements-and-anarchism [13.11.15]

21. Benjamin, W. (1999) The Arcades Project. Cambridge : Harvard University Press, K 3a1 395-396.

22. Foucault, M. (2001) Dits et écrits Tome I 1954-1975. Paris: Gallimard, texte n. 39.

23. Morin, M., Mezache, S. (2015) Saeio Nolens Volens. Paris : Galerie P38, catalogue d'exposition.

24. Cyop \& Kaf (2014) Taranto. Un anno in città vecchia. Napoli : Monitor.

\section{ABSTRACTS}

The polymorphic phenomenon commonly known as "street art " is an evident outcome of the pluralist and post-historical era of art. During the last ten years, it evolved in a way so we can no longer consider it as an isolated practice, characterized by independency and illegality; exclusively linked with a specific environment (the street), a geographic area or a specific media (the spraycan and the marker), nor it can be seen anymore as the expression of a pop aesthetics and culture. "Street art" has become a global practice, implying a wide range of techniques, media, styles and contents. Above all, it has gained several forms of recognition, coming from art institutions and market, city councils and the academic world. The present paper aims to propose a Danto-Hegelian interpretation of «street art ", according to which the phenomenon might have exhausted (if not « betrayed») its true aesthetic and ideological premises, thus going through a hardly definable metamorphosis.

Le phénomène polymorphe communément appelé «street art» est un digne fils de l'ère pluraliste et post-historique de l'art. Durant ces dix dernières années, il s'est développé d'une façon telle que, aujourd'hui, il ne nous apparaît plus comme une pratique isolée, indépendante, clandestine, exclusivement routière, géographiquement délimitée, faite à la bombe ou au feutre et relevant d'une esthétique pop. Le « street art » est devenu une pratique globale, impliquant un large éventail de techniques, médiums, sujets et styles. Surtout, il a fait l'objet de plusieurs 
formes de reconnaissance: de la part d'institutions artistiques, du marché de l'art, d'administrations citoyennes, de l'université. En proposant une lecture danto-hegelienne, cet article avance l'idée que le «street art " aurait désormais épuisé (sinon trahi) ses véritables fondements esthétiques et idéologiques, subissant une métamorphose au cadrage difficile.

INDEX

Chronological index: XXIe siècle

Mots-clés: street art, graffiti, fin de l'art, Arthur Danto, Blu

\section{AUTHOR}

\section{VITTORIO PARISI}

ATER / Doctorant, Université Paris 1 Panthéon-Sorbonne - Ufr 04 Arts plastiques et sciences de l'art, Institut ACTE Arts, Créations, Théorie, Esthétique - 47, rue des Bergers 75015 Paris. E-mail : . 